\title{
Fast visible imaging of ELM-wall interactions on JET
}

\author{
J.A. Alonso ${ }^{\mathrm{a}, *}$, P. Andrew ${ }^{\mathrm{b}}$, A. Neto ${ }^{\mathrm{c}}$, J.L. de Pablos ${ }^{\mathrm{a}}$, E. de la Cal ${ }^{\mathrm{a}}$, H. Fernandes ${ }^{\mathrm{c}}$, W. Fundamenski ${ }^{\mathrm{b}}$, \\ C. Hidalgo ${ }^{\text {a }}$, G. Kocsis ${ }^{\text {d }}$, A. Murari ${ }^{\text {e }}$, G. Petravich ${ }^{\text {d }}$, R.A. Pitts ${ }^{\text {f }}$, L. Rios ${ }^{\text {a }}$, C. Silva ${ }^{c}$, EFDA-JET contributors ${ }^{\text {g,1 }}$ \\ a Asociación EURATOM-CIEMAT, Av. Complutense 22, 28020 Madrid, Spain \\ ${ }^{\mathrm{b}}$ EURATOM-UKAEA Association, Culham Science Centre, Abingdom, UK \\ ${ }^{\mathrm{c}}$ Associação EURATOM-IST, Av. Rovisco Pais, 1049-001 Lisbon, Portugal \\ d EURATOM-HAS Association, KFKI-RMKI, Box 49, H1525 Budapest-114, Hungary \\ e Consorzio RFX-Associazione EURATOM ENEA per la Fusione, I-35127 Padova, Italy \\ ${ }^{\mathrm{f}}$ EURATOM-Confédération Suisse Association, EPFL, 1015 Lausanne, Switzerland \\ ${ }^{\mathrm{g}}$ JET-EFDA, Culham Science Centre, OX14 3DB, Abingdon, UK
}

\section{A R T I C L E I N F O}

\section{PACS:}

52.55.-S

52.55.Fa

\begin{abstract}
A B S T R A C T
We present and discuss recent measurements of the fast imaging diagnostic in JET. We focus on wide angle observations of few large ELM events $\left(\Delta W_{E L M} \sim 700 \mathrm{~kJ}\right)$ in plasmas with high current, magnetic field and stored energy ( $\sim 3 \mathrm{~T}, 3 \mathrm{MA}, 8 \mathrm{MJ})$. Measurements were taken at recording speeds around $30 \mathrm{kHz}$. The ELM-wall interaction shows a filamentary structure that appears together with the radiation increase in the divertor, implying similar times of flight for ELM radial and parallel transport in the SOL, $\tau_{\|} \sim \tau_{\mathrm{r}}$, within a time resolution of $30 \mu \mathrm{s}$. Two high and low triangularity cases are compared. For high triangularity the ELM-wall interaction is seen to start in the upper dump plate reaching the outer limiters some $70 \mu$ s later. In the low triangularity cases interaction generally starts in the outer wall but clear evidence of radiation increase in the upper dump plate is seen shortly after $(30 \mu \mathrm{s})$ or together with the first filament impact on the outer limiters. A poloidal drift of the filament strike point on the limiters is sometimes visible which allows an estimation of the perpendicular filament velocity $(\sim 1 \mathrm{~km} / \mathrm{s})$.
\end{abstract}

(ㄷ) 2009 Elsevier B.V. All rights reserved.

\section{Introduction}

Fast plasma visible imaging is becoming a common technique for studying plasma boundary phenomena. Edge turbulence $[9,1]$, blob propagation in the SOL [4], L-mode filament dynamics [2] and Edge Localised Mode structure [5] have been investigated by means of fast visible cameras. The understanding of the ELM drive and energy exhaust mechanisms is particularly important from an ITER perspective as they can inflict high power densities on plasma facing components limiting their lifetime and risking the safe operation of $\mathrm{H}$-mode plasmas. Experimental validation of existing ELM [8] and filament energy transport [3] theories is therefore necessary to asses the design of mitigation techniques and first wall materials. In particular, to predict the maximum local, transient heat loads on the divertor plates and limiter tiles, the role of the filamentary structures observed during the development of the ELM instability, their energy content and their radial and parallel dynamics has to be clarified.

\footnotetext{
* Corresponding author.

E-mail address: arturo.alonso@jet.uk (J.A. Alonso).

1 See the Appendix of M.L.Watkins et al., Fusion Energy 2006 (Proceedings 21st International Conference Chengdu, 2006) IAEA, 2006.
}

Fast imaging can be a useful diagnostic to help answering these and other questions. Such efforts have very recently begun on JET with the installation of a Photron APX fast camera viewing the full poloidal cross-section of the vacuum vessel and covering a toroidal extent of $\sim 90^{\circ}$. This wide view setup is suited for the study of large scale instabilities. Here, we study measurements recorded in a dedicated session designed to investigate the physics of large ELMs using plasmas in a vertical target, low triangularity $(\delta \sim 0.25)$ configuration at high current $\left(I_{p}=3.0 \mathrm{MA}, B_{\phi}=3.0 \mathrm{~T}\right)$, high heating power $\left(P_{N B I}=15-20 \mathrm{MW}\right)$ and plasma stored energy $(\mathrm{W} \sim 8 \mathrm{MJ})$ (see Ref. [6] for more details and results on these experiments). For the sake of comparison, a high triangularity $(\delta \sim 0.40)$ case is presented the plasma parameters being otherwise similar (see Fig. 1).

\section{Observations and estimations}

A few events have been recorded with $\Delta W_{E L M}$ approaching $1 \mathrm{MJ}$. Camera images have been obtained in visible unfiltered light at $30 \mathrm{kfps}$ (33 $\mathrm{s}$ inter-frame) and with $20 \mu \mathrm{s}$ integration time. Field-aligned plasma filaments are visible in the fast camera images as bright spots on the outboard poloidal limiters and on the upper dump plates (see Figs. 3 and 5). Timing issues in the 


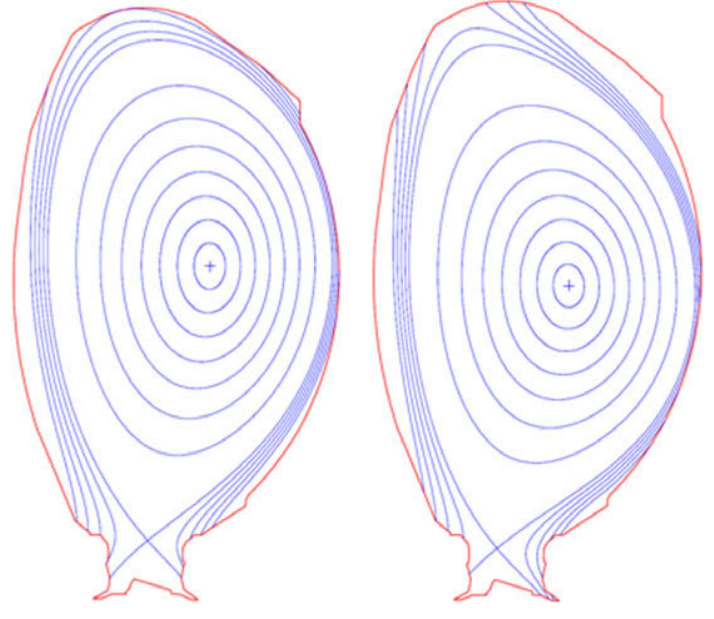

Fig. 1. Magnetic configurations for the discharges in Figs. 2-6. Left: JET pulse $\# 70228$ at 60.7 s. Right: JET pulse \#69903 at 58.5 s.

hardware prevented absolute comparison of the new camera data on a fast timescale with other diagnostic signals but, as the camera observes both regions simultaneously, it is clear (Fig. 3) that the filament interaction with the main chamber surfaces is coincident with the very start of radiation increase in the lower divertor region. If one assumes that filaments were born at the very beginning of the transport barrier breakdown coincident with the start of the parallel streaming of energy and particles from somewhere near the outer mid plane to the divertor, this observation implies a comparable radial and parallel characteristic transport times and therefore of a radial velocity of the order $v_{r} / c_{s} \approx \Delta r / \mathrm{L}_{\|}$, where $\Delta r$ is the plasma-wall clearance distance. Interestingly, for the two ELMs in Figs. 2 and 3 interaction with the upper dump plate $(\Delta r=20 \mathrm{~cm}$;

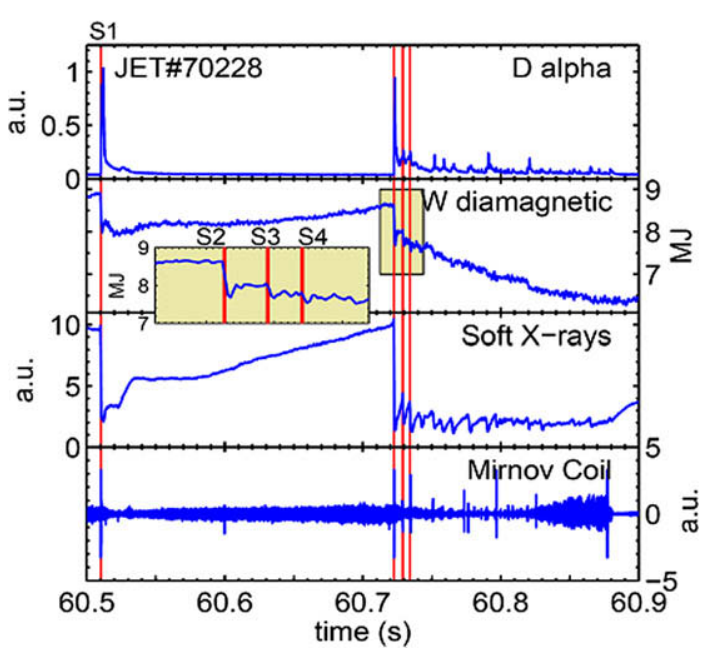

Fig. 2. Time traces of the evolution of two Type-I ELMs the second being a compound ELM. Vertical red lines mark the time lapse of the image sequences in Fig. 3. (For interpretation of the references to color in this figure legend, the reader is referred to the web version of this article).

$4 \mathrm{~cm}$ mapped at the outer mid plane) and outer limiters $(\Delta r=2-$ $3 \mathrm{~cm}$ ) start at the same time casting a radial velocity in the range of few $100 \mathrm{~m} / \mathrm{s}$, consistent with the effective velocity of Langmuir probe ExB flux measurements [7]. From our measurements and after inspecting the magnetic equilibrium we cannot confidently say whether the early interaction with the upper dump plate is due to the radial (upwards) propagation of a filament or to the parallel flux of the energy transported to the far SOL by the filaments observed in the outer mid-plane. The subsequent energy drops and $D_{\alpha}$ spikes of the second compound ELM in Fig. 2 are seen to be

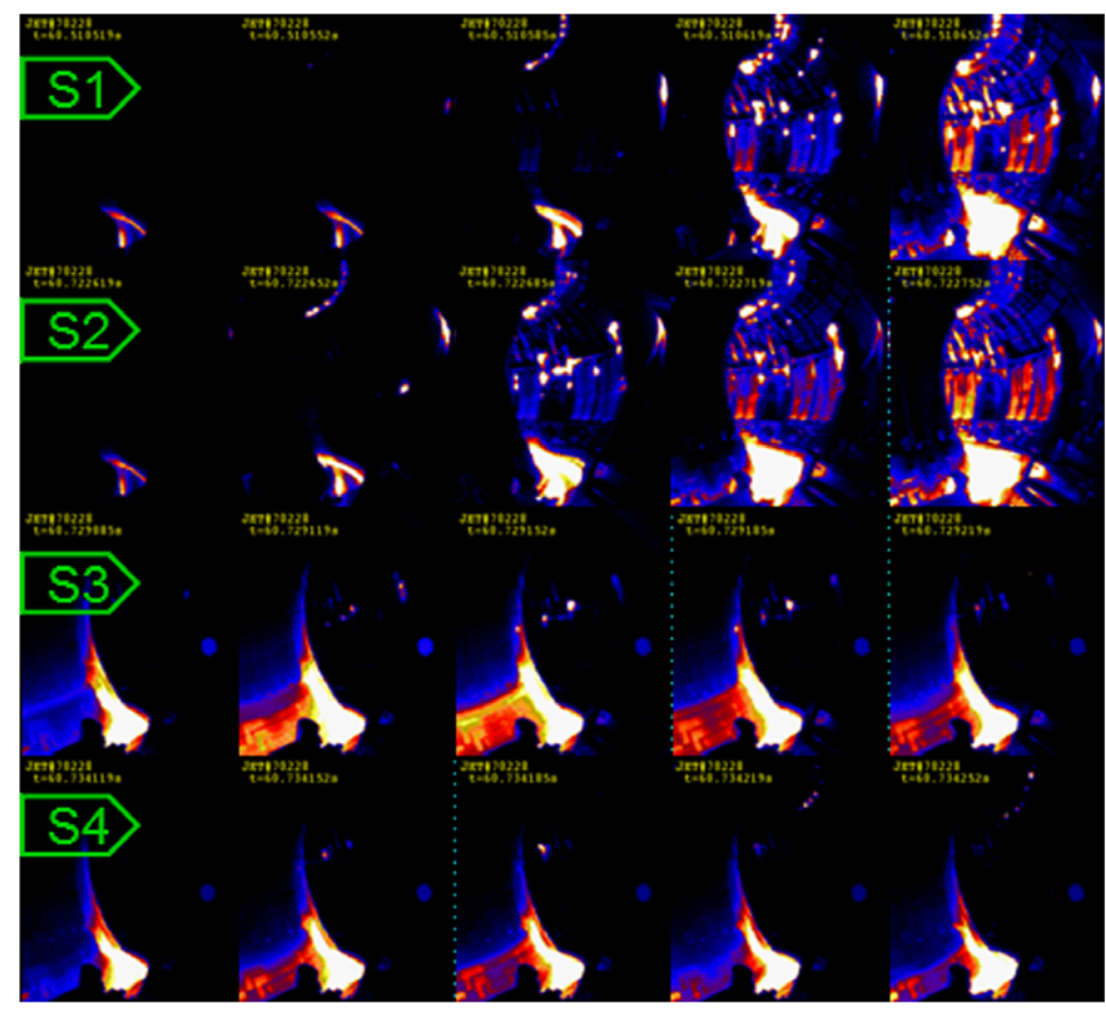

Fig. 3. Image sequences for the times in Fig. $2 . \mathrm{S} 1$ and $\mathrm{S} 2$ correspond to the ELM initial development. S3 and S4 show the aftershock activity of a compound ELM. 


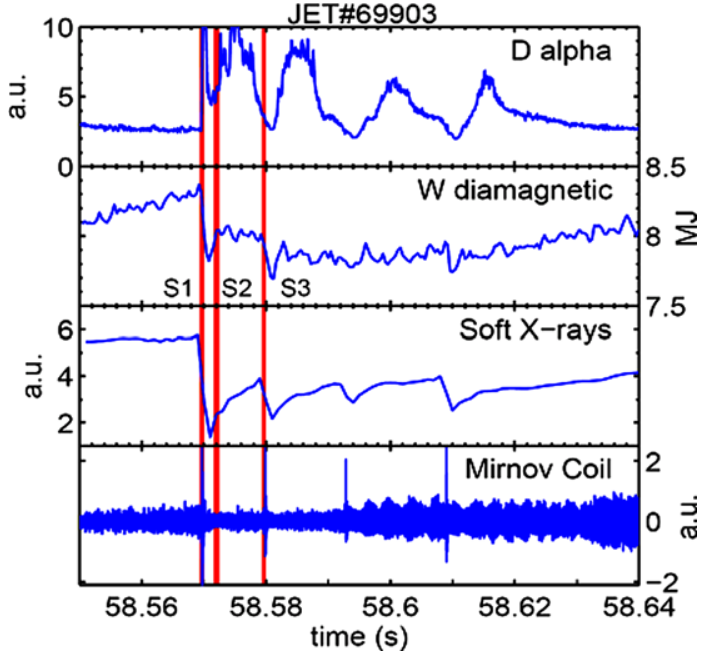

Fig. 4. Time traces of the evolution of one Type-I ELM. Vertical red lines mark the time lapse of the image sequences in Fig. 5. (For interpretation of the references to color in this figure legend, the reader is referred to the web version of this article).

associated with a radiating belt in the inner divertor and filaments appearing on the outer mid plane and close to the upper plate (see Fig. 3). For these ELMs, filaments are evident in the images for a fraction $(\sim 400 \mu \mathrm{s})$ of the diamagnetic energy drop period $(\sim 2-$ $3 \mathrm{~ms}$ ). IR thermography reports on energy deposition in the outboard limiter of about $10 \%$ of the ELM energy for these large ELM discharges [6].

Figs. 4 and 5 show a Type-I ELM ( $\left.\Delta W_{E L M} \sim 400 \mathrm{~kJ}\right)$ observed in a high triangularity configuration. ELM-wall interaction is observed first at the upper dump plate and at the lower part of the outer wall (although this can be due to the higher neutral particle density near the divertor). Two frames ( $70 \mu \mathrm{s})$ later, filaments are found in the outer mid plane. MARFE-like phenomena are commonly observed in high triangularity discharges. The high parallel energy fluxes reaching the upper plates increase recycling and neutral desorption (Fig. 5). A highly radiating neutral cloud is then dragged along the field lines around the central column until it reaches the inner divertor region where a radiating belt is also observed. It is there where most of the ELM radiation power fraction is localised. As shown in Fig. 5, the ELM aftershocks present an interaction pattern similar to the first ELM strike. For these ELM events, filament interaction with the wall is observed for most of the energy release time and their footprints on the limiters are seen to drift poloidaly (Fig. 6). As the parallel motion of the particles in the filament is unobservable one can only speak about the perpendicular (i.e. in the diamagnetic direction) motion of the filament. Using the average safety factor value in the sepatratrix $(q=5)$ as the filament tilting one obtains a perpendicular velocity of $1 \mathrm{~km} / \mathrm{s}$. Estimates of toroidal or poloidal velocities can only be obtained under particular assumptions on the neat filament velocity.

\section{Conclusions}

First fast camera measurements of large ELM events on JET have been presented. We have reported on the observation of clear, field aligned plasma filaments visible on the images as aligned bright spots on the outer limiters and upper dump plate. The interaction with the later is remarkable not only in the high triangularity case studied but also in some large ELM instances in low triangularity configurations where it is observed shortly after or together with the first filament impacts on the outer limiters. For the shots studied here, the ELM filament radial velocity was estimated to be a few $100 \mathrm{~m} / \mathrm{s}$. In a few cases, the ELM filaments could be tracked along the surface of the outer limiters, allowing the estimation of their perpendicular velocity, found to be $\sim 1000 \mathrm{~m} / \mathrm{s}$. The observation of compound ELM aftershocks also revealed filamentary structures. In high triangularity configurations the high fluxes on the upper dump plate are seen to cause the desorbtion of neutral gas seen as a highly radiating MARFE-like cloud that is dragged along the field line around the central column until the inner divertor region.

\section{Acknowledgements}

This work, supported by the European Communities under the contract of Association between EURATOM-CIEMAT, was carried out within the framework of the European Fusion Development

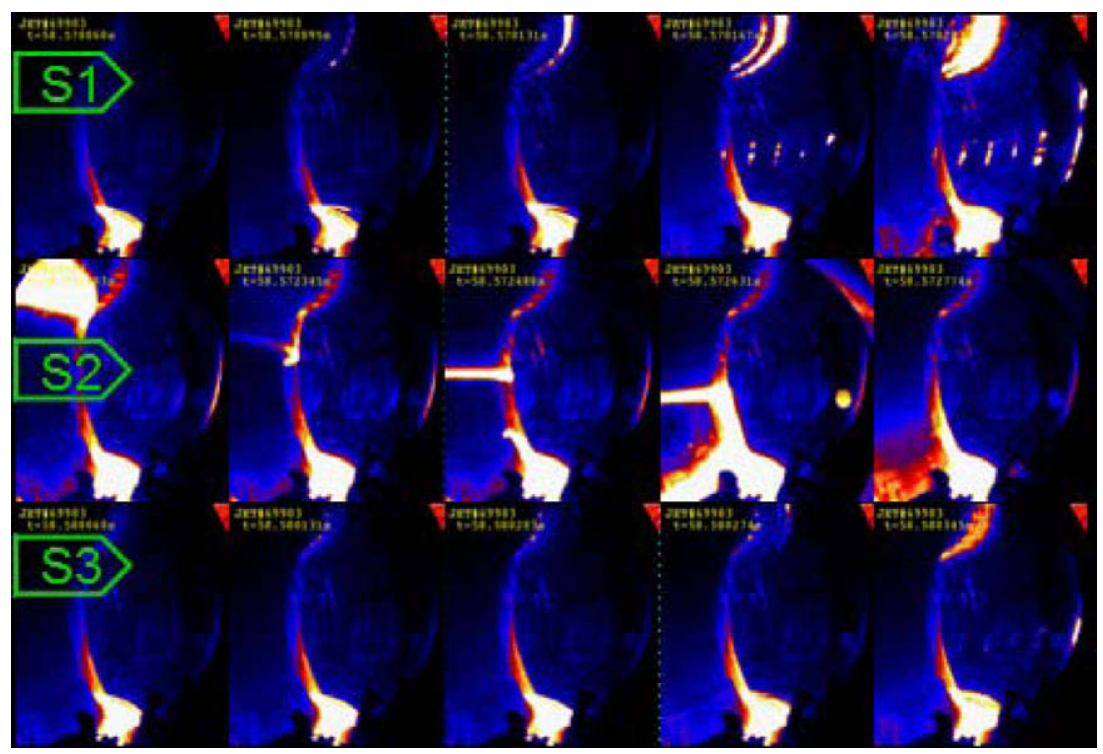

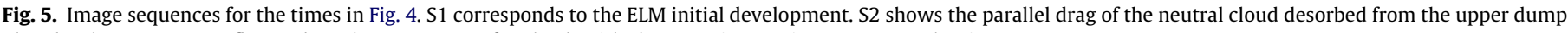
plate by the ELM energy flux and S3 shows an ELM aftershock with the same interaction pattern as that in S1. 


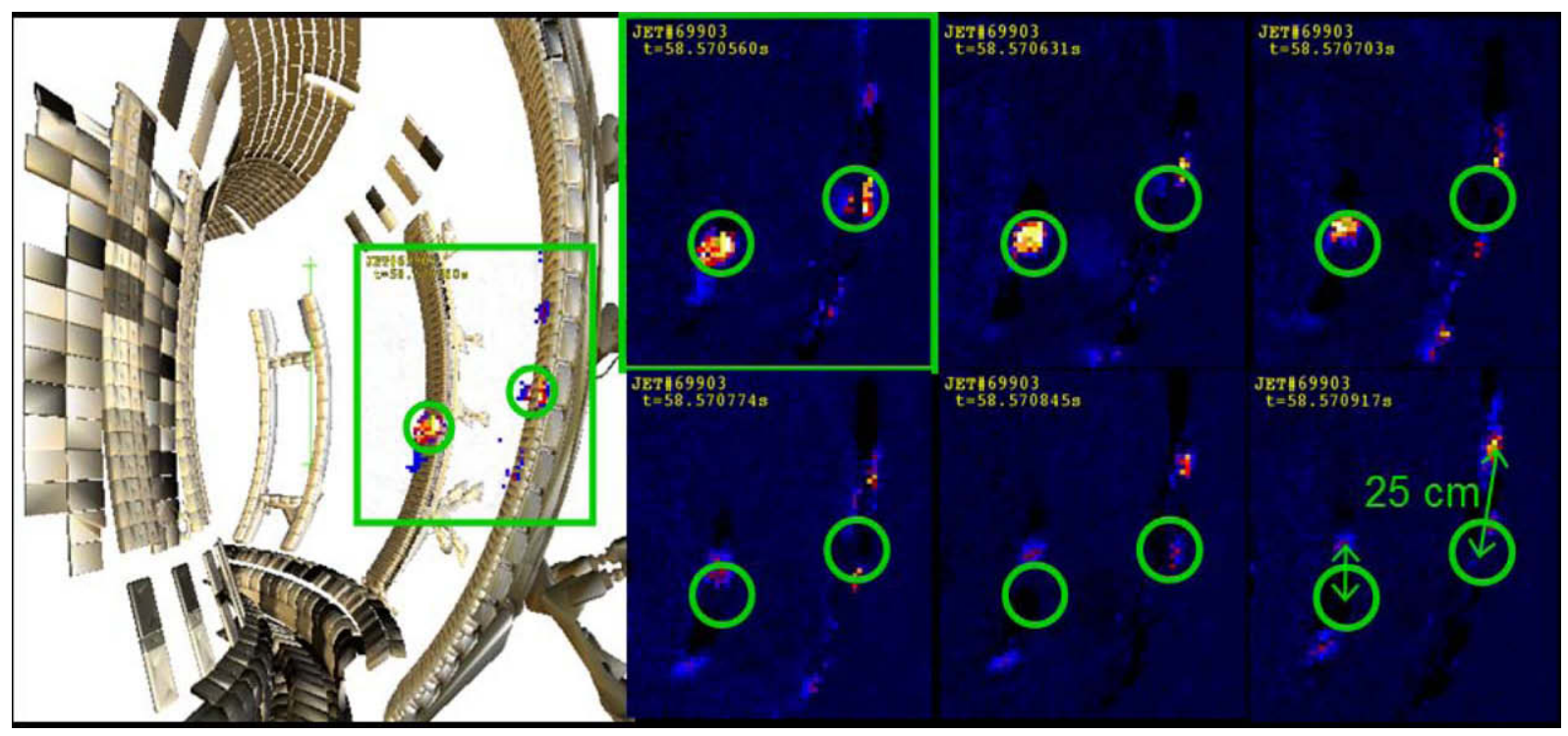

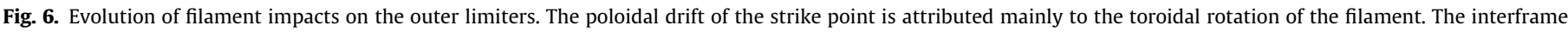
separation is $71 \mu \mathrm{s}$ (displaying one every two frames). The circles are fixed on the initial position of the interaction point.

Agreement. The views and opinions expressed herein do not necessarily reflect those of the European Commission.

\section{References}

[1] J.A. Alonso, S.J. Zweben, et al., Plasma Phys. Control. Fusion 48 (12B) (2006) B465.

[2] N.B. Ayed et al., In: Proceedings of the 34th EPS conference on Control. Fusion and Plasma Physics, Warsaw, Polland, 2007.
[3] W. Fundamenski, R.A. Pitts, Plasma Phys. Control. Fusion 48 (2006) 109.

[4] O. Grulke, J.L. Terry, et al., Phys. Plasmas 13 (1) (2006) 012306.

[5] A. Kirk, H.R. Wilson, et al., Phys. Rev. Lett. 92 (24) (2004) 245002

[6] R. Pitts et al., J. Nucl. Mater. 390-391 (2009) 755

[7] C. Silva et al., J. Nucl. Mater. 390-391 (2009) 355

[8] H.R. Wilson, S.C. Cowley, et al., Plasma Phys. Control. Fusion 48 (2006) A71.

[9] S.J. Zweben, R.J. Maqueda, et al., Nucl. Fusion 44 (1) (2004) 134. 